Research Paper

\title{
Perineural and lymphovascular invasion predicts for poor prognosis in locally advanced rectal cancer after neoadjuvant chemoradiotherapy and surgery
}

\author{
Quanquan Sun ${ }^{1,2,}$, Tongxin Liu1,2, ${ }^{1,}$, Peng Liu1,2, Jialin Luo ${ }^{1,2}$, Na Zhang1,2, Ke Lu1,2, Haixing Ju3, Yuping \\ $\mathrm{Zhu}^{3}$, Wei Wu${ }^{4}$, Lingnan Zhang ${ }^{5}$, Yongtian Fan ${ }^{3}$, Yong Liu ${ }^{3}$, Dechuan $\mathrm{Li}^{3}$, Yuan $\mathrm{Zhu}^{1,2}$, Luying Liu ${ }^{1,2}$ \\ 1. Department of Radiation Oncology, Zhejiang Cancer Hospital, Hangzhou, Zhejiang Province, China \\ 2. Zhejiang Key Laboratory of Radiation Oncology, Zhejiang Cancer Hospital, Hangzhou, Zhejiang Province, China \\ 3. Department of Colorectal Surgery, Zhejiang Cancer Hospital, Hangzhou, Zhejiang Province, China \\ 4. Department of Pathology, Zhejiang Cancer Hospital, Hangzhou, Zhejiang Province, China \\ 5. Department of Radiology, Zhejiang Cancer Hospital, Hangzhou, Zhejiang Province, China \\ * These authors contributed equally to this work \\ $\square$ Corresponding author: Prof. Luying Liu, Department of Radiation Oncology, Zhejiang Cancer Hospital, Banshan Road East, Hangzhou 310022, Zhejiang, \\ China. Email: luyingliu@163.com; and Yuan Zhu, Email: zhuyuan63@hotmail.com \\ (c) Ivyspring International Publisher. This is an open access article distributed under the terms of the Creative Commons Attribution (CC BY-NC) license \\ (https:// creativecommons.org/licenses/by-nc/4.0/). See http://ivyspring.com/terms for full terms and conditions.
}

Received: 2018.11.13; Accepted: 2019.03.30; Published: 2019.05.21

\begin{abstract}
Background: Perineural invasion (PNI) and lymphovascular invasion (LVI) are associated with poor prognosis in colorectal cancer, but their clinical significance is still controversial for patients with locally advanced rectal cancer (LARC) who had received neoadjuvant chemoradiotherapy (nCRT) and surgical resection. The aim of this study was to confirm the correlation between PNI and/or LVI and clinical prognosis and to further confirm whether PNI and/or LVI can be used as potential prognostic indicators of adjuvant chemotherapy after $\mathrm{nCRT}$ and surgery in LARC.

Methods: From February 2002 to December 2012, a total of 181 patients with LARC who had received $\mathrm{nCRT}$ and surgical resection were retrospectively reviewed. Overall survival (OS) and disease-free survival (DFS) were determined by the Kaplan-Meier method, log-rank test, and Cox proportional hazard regression model.

Results: The median follow-up time was 48 months (range, 3 to 162 months). All the PNI-positive and/or LVI-positive patients showed adverse DFS and OS $(P<0.001)$. In multivariate analysis, $\mathrm{PNI}$ and LVI were independent prognostic factors for DFS. PNI, rather than LVI, was also an independent prognostic factor for OS. In a subgroup analysis, PNI-positive, rather than LVI-positive, may benefit from adjuvant chemotherapy.

Conclusion: For patients with LARC undergoing nCRT and surgery, PNI-positive and/or LVI positive were associated with poorer DFS and OS. And PNI-positive, rather than LVI-positive, may benefit from adjuvant chemotherapy.
\end{abstract}

Key words: locally advanced rectal cancer, perineural invasion, lymphovascular invasion, neoadjuvant therapy

\section{Introduction}

Preoperative neoadjuvant chemoradiotherapy (nCRT) followed by total mesorectal excision (TME) was the standard of care for locally advanced rectal cancer (LARC, T3-4/N+) in the past decade $[1,2]$. Fluorouracil-based postoperative adjuvant chemotherapy has been recommended for all patients undergoing preoperative chemoradiotherapy by National Comprehensive Cancer Network (NCCN) guidelines [3]. However, the benefit of adjuvant chemotherapy for all patients has been controversial over the past few years. EORTC 22921 trial demonstrated no significant benefit for chemotherapy 
in LARC after nCRT or preoperative radiation alone [4]. Other studies reported significantly increased survival with postoperative chemotherapy [5]. ESMO guidelines suggested that the decision of postoperative chemotherapy should balance the risk of relapse for a particular patient and the predicted toxicity [6]. Thus, identifying the certain subsets of patients who may benefit from adjuvant chemotherapy will help to optimize the modality of treatment as well as diminish the toxicities caused by chemotherapy.

Two pathologic features that have been shown to be correlated with outcome in CRC are perineural invasion (PNI) and lymphovascular invasion (LVI). PNI is a pathologic process of tumor invasion of nervous structures, and it spreads along nerve sheaths [7]. LVI is defined as tumor cells invasion in the small lymphatic or blood vessels [8]. Studies have indicated that both of these pathologic features are the prognostic factors in rectal cancer [9-11]. Rectal cancer patients with PNI or LVI positive usually have a high incidence of local recurrence and distant metastasis $[11,12]$. However, the role of these two pathologic variables in predicting the prognosis of LARC patients has been still controversial. Several studies demonstrated that PNI or LVI is not related markedly to the poor prognosis after curative resection of colorectal cancers [13,14]. In this study, we retrospectively evaluated the clinical significance of PNI and LVI as indicators of adjuvant chemotherapy after nCRT and surgery in LARC.

\section{Methods}

\section{Patient selection}

The records of 204 consecutive patients with LARC (clinically T3-4 and/or node positive) who underwent nCRT followed by surgical resection at the Zhejiang Cancer Hospital between February 2002 and December 2012 were retrospectively reviewed. Data pertaining to demographics, staging, tumor markers, pathology, treatment, and outcomes were collected for each patient. Twelve patients were removed because metastatic cancer was found during the course of surgery. Eleven patients were excluded due to the limited follow-up information. Finally, a total of 181 patients were available for analysis. This study was approved by the Independent Ethics Committee of Zhejiang Cancer Hospital.

\section{Staging and treatment}

All patients were restaged according to the AJCC 2010 staging system. Chest, abdominal, and pelvic CT scan, pelvic MRI with contrast or endorectal ultrasound were performed for tumor, nodal, and metastasis staging. The main neoadjuvant for chemotherapy was 5-Fu or capecitabine-based chemotherapeutic regimen. A dose of 40-45 Gy radiotherapy was delivered to the whole pelvis in 1.8 Gy daily fractions, followed by a 5.4 Gy boost to the primary tumor in 3 fractions. Radical proctectomy, including abdominoperineal resection (APR), low anterior resection (LAR), and Hartmann's procedure, was performed 6 to 10 weeks after completion of nCRT. XELOX or FOLFOX6 postoperative adjuvant chemotherapeutic regimen was recommended for all patients 4 weeks after surgery. However, only 102 patients $(56.4 \%)$ received adjuvant chemotherapy. Due to postoperative complications, economic problems or other reasons, the other 79 patients $(43.6 \%)$ did not receive adjuvant chemotherapy.

\section{Histopathologic analysis}

For each patient included in this study, standard pathologic analysis by a pathologist with expertise in colorectal cancer was performed on all surgical specimens. Pathologic features, such as tumor size, tumor infiltration, number of positive lymph nodes, histologic grade, extranodal extension, PNI, LVI, as well as other features included in the standard protocol were documented. The widely accepted definition of PNI-positive is inclusive of two aspects: at least one-third of the nerve is surrounded by tumor cells without invasion of the nerve sheath (SS-PNI), as well as any of the three layers of the nerve sheath is invaded by tumor cells (TS-PNI) $[15,16]$. Studies have indicated that patients in the SS-PNI subgroup and TS-PNI subgroup have a similar 5-year local recurrence rate [17]. In our study, both SS-PNI and TS-PNI were defined as PNI-positive. LVI was defined as tumor invasion in the vascular and lymphatic structure. Since it is infeasible to distinguish histologically between lymphatic and venous vessels, the term LVI usually refers to any of these structures [18].

\section{Statistical analysis}

Associations between clinical and pathological characteristics of the tumor were assessed by the chi-square test. Disease-free survival (DFS) was defined as the time from the completion of surgery to any relapse or death. Overall survival (OS) was calculated from the date of diagnosis to the date of death. DFS was censored at the time of the last follow-up for disease-free or non-cancer-related death patients. OS was censored at the time of the last follow-up for alive patients. The influence of PNI or LVI on DFS and OS was conducted using the Kaplan-Meier survival analysis and log-rank tests. Multivariate analysis was performed using Cox proportional hazard regression. A $P$-value $<0.05$ was 
considered statistically significant. All statistical analyses were performed using SPSS 13.0 software (SPSS, Chicago, IL).

\section{Results}

\section{Patient characteristics}

The characteristics of the 181 LARC patients are shown in Table 1. The median follow-up time was 48 months (range, 3 to 162 months). Median patient age at the time of diagnosis was 51-year-old (range, 23 to 76-year-old). In our cohort, 44 patients (24.3\%) and 137 patients $(75.7 \%)$ were PNI positive and PNI negative, respectively, and 26 patients $(14.4 \%)$ and 155 patients $(85.6 \%)$ were LVI positive and negative, respectively. There were statistically significant differences in nodal status between PNI+ and PNIgroups $(P=0.033)$ or LVI+ and LVI- groups $(P=0.020)$. No significant differences were observed in other demographic and treatment-related characteristics of PNI or LVI groups.

Table 1. Clinical and Treatment-related Characteristics by PNI and LVI status

\begin{tabular}{|c|c|c|c|c|c|c|}
\hline Characteristic & $\begin{array}{l}\text { PNI+ } \\
\mathrm{N}=44 \\
(24.3 \%)\end{array}$ & $\begin{array}{l}\text { PNI- } \\
\text { N=137 } \\
(75.7 \%)\end{array}$ & $P$ & $\begin{array}{l}\text { LVI+ } \\
\text { N=26 } \\
(14.4 \%)\end{array}$ & $\begin{array}{l}\text { LVI- } \\
\mathrm{N}=155 \\
(85.6 \%)\end{array}$ & $P$ \\
\hline $\begin{array}{l}\text { Age (median, } \\
\text { 51) }\end{array}$ & & & 0.153 & & & 0.193 \\
\hline$\leq 51$ & $18(40.9)$ & 73 (53.3) & & $10(38.5)$ & 81 (52.3) & \\
\hline$>51$ & $26(59.1)$ & 64 (46.7) & & $16(61.5)$ & 74 (47.7) & \\
\hline Sex & & & 0.603 & & & 0.826 \\
\hline Male & $30(68.2)$ & 99 (72.3) & & 19 (73.1) & $110(71.0)$ & \\
\hline Female & $14(31.8)$ & $38(27.7)$ & & $7(26.9)$ & $45(29.0)$ & \\
\hline Tumor location & & & 0.860 & & & 0.776 \\
\hline$\leq 5 \mathrm{~cm}$ & $26(59.1)$ & $83(60.6)$ & & 15 (57.7) & 94 (60.6) & \\
\hline$>5 \mathrm{~cm}$ & $18(40.9)$ & $54(39.4)$ & & $11(42.3)$ & $61(39.4)$ & \\
\hline CEA (ng/ml) & & & 0.768 & & & 0.524 \\
\hline$\leq 5$ & $22(50.0)$ & $72(52.6)$ & & $12(46.2)$ & $82(52.9)$ & \\
\hline$>5$ & $22(50.0)$ & $65(47.4)$ & & $14(53.8)$ & 73 (47.1) & \\
\hline $\begin{array}{l}\text { Radiation dose } \\
\text { (Gy) }\end{array}$ & & & 0.685 & & & 0.905 \\
\hline$\leq 45.0$ & $3(6.8)$ & $12(8.8)$ & & $2(7.7)$ & $13(8.4)$ & \\
\hline$>45.0$ & 41 (93.2) & $125(91.2)$ & & $24(92.3)$ & $142(91.6)$ & \\
\hline Tumor stage & & & 0.537 & & & 0.053 \\
\hline $\mathrm{T} 3$ & $16(36.4)$ & 57 (41.6) & & $6(23.1)$ & $67(43.2)$ & \\
\hline $\mathrm{T} 4$ & $28(63.6)$ & $80(58.4)$ & & $20(76.9)$ & $88(56.8)$ & \\
\hline Nodal status & & & 0.033 & & & 0.020 \\
\hline Negative & $15(34.1)$ & $72(52.6)$ & & 7 (26.9) & $80(51.6)$ & \\
\hline Positive & $29(65.9)$ & 65 (47.4) & & $19(73.1)$ & 75 (48.4) & \\
\hline $\begin{array}{l}\text { Adjuvant } \\
\text { Chemotherapy }\end{array}$ & & & 0.119 & & & 0.238 \\
\hline No & $22(50.0)$ & 57 (41.6) & & $15(57.7)$ & $64(41.3)$ & \\
\hline XELOX & $18(40.9)$ & $48(35.0)$ & & $6(23.1)$ & $60(38.7)$ & \\
\hline FOLFOX & $4(9.1)$ & $32(23.4)$ & & 5 (19.2) & $31(20.0)$ & \\
\hline
\end{tabular}

\section{Pathologic characteristics after neoadjuvant chemoradiotherapy}

Pathologic features of our cohort are listed in Table 2 . There were statistically significant differences in ypT stage, ypN stage, AJCC stage, and resection level completeness between PNI+ and PNI- groups or LVI+ and LVI- groups (all $P<0.05$ ). We observed a statistically significant difference between PNI+ and PNI- groups, rather than LVI+ or LVI- groups, with regard to tumor differentiation $(P=0.002)$. There were no statistically significant differences in pathologic type between PNI or LVI groups.

Table 2. Pathologic Features After Neoadjuvant Chemoradiation by $\mathrm{PNI}$ and LVI status

\begin{tabular}{|c|c|c|c|c|c|c|}
\hline Characteristic & $\begin{array}{l}\text { PNI+ } \\
\text { N=44 } \\
(24.3 \%)\end{array}$ & $\begin{array}{l}\text { PNI- } \\
\mathbf{N}=137 \\
(75.7 \%)\end{array}$ & $P$ & $\begin{array}{l}\text { LVI+ } \\
N=26 \\
(14.4 \%)\end{array}$ & $\begin{array}{l}\text { LVI- } \\
\mathrm{N}=155 \\
(85.6 \%)\end{array}$ & $P$ \\
\hline ypT stage & & & $<0.001$ & & & 0.001 \\
\hline T0-2 & $5(11.4)$ & $71(51.8)$ & & $3(11.5)$ & 73 (47.1) & \\
\hline T3-4 & $39(88.6)$ & $66(48.2)$ & & $23(88.5)$ & $82(52.9)$ & \\
\hline ypN stage & & & $<0.001$ & & & $<0.001$ \\
\hline N0 & $6(13.6)$ & $95(69.3)$ & & $4(15.4)$ & $97(62.6)$ & \\
\hline N1 & $24(54.5)$ & $31(22.6)$ & & $12(46.2)$ & $43(27.7)$ & \\
\hline N2 & $14(31.8)$ & $11(8.0)$ & & $10(38.5)$ & $15(9.7)$ & \\
\hline AJCC stage & & & $<0.001$ & & & $<0.00$ \\
\hline 0 & $0(0.0)$ & $37(27.0)$ & & $0(0.0)$ & $37(23.9)$ & \\
\hline 1 & $0(0.0)$ & $20(14.6)$ & & $0(0.0)$ & $20(12.9)$ & \\
\hline 2 & $7(15.9)$ & $38(27.7)$ & & $4(15.4)$ & $41(26.5)$ & \\
\hline 3 & $37(84.1)$ & $42(30.7)$ & & $22(84.6)$ & $57(36.8)$ & \\
\hline Pathologic type & & & 0.756 & & & 0.466 \\
\hline Adenocarcinoma & $37(84.1)$ & $118(86.1)$ & & $22(84.6)$ & $133(85.8)$ & \\
\hline $\begin{array}{l}\text { Mucinous } \\
\text { adenocarcinoma }\end{array}$ & $6(13.6)$ & $14(10.2)$ & & $4(15.4)$ & $16(10.3)$ & \\
\hline $\begin{array}{l}\text { Signet-ring cell } \\
\text { carcinoma }\end{array}$ & $1(2.3)$ & $5(3.6)$ & & $0(0.0)$ & $6(3.9)$ & \\
\hline Differentiation & & & 0.002 & & & 0.081 \\
\hline Well & $0(0.0)$ & $3(2.2)$ & & $0(0.0)$ & $3(1.9)$ & \\
\hline Moderately & $21(47.7)$ & $76(55.5)$ & & $11(42.3)$ & $86(55.5)$ & \\
\hline Poorly & $23(52.3)$ & $37(27.0)$ & & $14(53.8)$ & $46(29.7)$ & \\
\hline Other & $0(0.0)$ & $21(15.3)$ & & $1(3.8)$ & $20(12.9)$ & \\
\hline $\begin{array}{l}\text { Resection level } \\
\text { completeness }\end{array}$ & & & 0.011 & & & 0.041 \\
\hline R0 & $40(90.9)$ & $136(99.3)$ & & $24(92.3)$ & $152(98.1)$ & \\
\hline R1 & $3(6.8)$ & $1(0.7)$ & & $1(3.8)$ & $3(1.9)$ & \\
\hline R2 & $1(2.3)$ & $0(0.0)$ & & $1(3.8)$ & $0(0.0)$ & \\
\hline
\end{tabular}

\section{Role of PNI and LVI as prognostic factors of outcome in LARC patients}

Univariate analyses were performed to investigate the prognostic significance of PNI and LVI as well as other pathologic and clinical variables. As shown in Table 3, PNI or LVI positive was significantly associated with poorer DFS and OS (all $P$-value $<0.001)$. In addition, patients with ypT3-4 stage had poorer DFS $(P<0.001)$ and OS $(P<0.001)$. Likewise, patients with positive $\mathrm{pN}$ status also had poorer DFS $(P<0.001)$ and OS $(P<0.001)$. The other variables investigated in our univariate analysis did not significantly affect the outcome.

The 3-year DFS rate was $76.8 \%$ for PNI negative patients and $26.2 \%$ for PNI positive patients $(P<0.001$, Figure 1A, Left). The 3-year OS rate was $82.8 \%$ for PNI negative patients and $31.0 \%$ for PNI positive patients $(P<0.001$, Figure 1B, Left). Similar results were found in LVI groups. Patients with LVI negative status 
exhibited a two-fold increase in 3-year DFS and OS compared with patients with LVI positive status ( $71.2 \%$ vs. $25.0 \%$ and $75.5 \%$ vs. $38.3 \%$, respectively; $P<0.001$, Figure $1 \mathrm{~A}$ and $1 \mathrm{~B}$, Right). However, patients with both PNI and LVI positive did not get a further worse outcome. The 3-year DFS rate was $33.6 \%$ for PNI-LVI+ or PNI+LVI- patients and $18.9 \%$ for both PNI and LVI positive patients ( $P=0.126$, Figure 2A). The 3-year OS rate was $36.8 \%$ for PNI-LVI+ or PNI+LVI- patients and $31.2 \%$ for both PNI and LVI positive patients ( $P=0.106$, Figure $2 \mathrm{~B})$. The 3 -year DFS and OS for both PNI and LVI negative patients were $78.8 \%$ and $84.1 \%$, respectively (Figure 2 ).

Table 3. Univariate analysis for disease-free and overall survival

\begin{tabular}{|c|c|c|c|c|}
\hline \multirow{2}{*}{$\begin{array}{l}\text { Univariate } \\
\text { analysis }\end{array}$} & \multicolumn{2}{|c|}{ Disease-free survival } & \multicolumn{2}{|l|}{ Overall survival } \\
\hline & HR (95\% CI) & $P$ & HR (95\% CI) & $P$ \\
\hline \multicolumn{5}{|l|}{ PNI } \\
\hline $\begin{array}{l}\text { Positive vs. } \\
\text { negative }\end{array}$ & $4.865(3.023-7.831)$ & $<0.001$ & $6.350(3.852-10.467)$ & $<0.001$ \\
\hline \multicolumn{5}{|l|}{ LVI } \\
\hline $\begin{array}{l}\text { Positive vs. } \\
\text { negative }\end{array}$ & $4.506(2.738-7.413)$ & $<0.001$ & $4.634(2.808-7.645)$ & $<0.001$ \\
\hline \multicolumn{5}{|l|}{ ypT stage } \\
\hline T0-2 vs. T3-4 & $3.556(2.042-6.191)$ & $<0.001$ & $4.950(2.595-9.441)$ & $<0.001$ \\
\hline \multicolumn{5}{|l|}{ ypN stage } \\
\hline N0 vs. N+ & 3.767 (2.300-6.170) & $<0.001$ & $5.079(2.972-8.679)$ & $<0.001$ \\
\hline \multicolumn{5}{|l|}{ Tumor location } \\
\hline$\leq 5 \mathrm{~cm}$ vs. $>5 \mathrm{~cm}$ & $1.189(0.752-1.880)$ & 0.458 & $1.220(0.757-1.964)$ & 0.414 \\
\hline \multicolumn{5}{|l|}{ CEA (ng/ml) } \\
\hline$\leq 5$ vs. $>5$ & $1.359(0.862-2.141)$ & 0.186 & 1.449 (0.901-2.331) & 0.126 \\
\hline \multicolumn{5}{|l|}{$\begin{array}{l}\text { Adjuvant } \\
\text { Chemotherapy }\end{array}$} \\
\hline Yes vs. No & 0.758 (0.481-1.193) & 0.231 & $0.702(0.437-1.129)$ & 0.144 \\
\hline
\end{tabular}

A

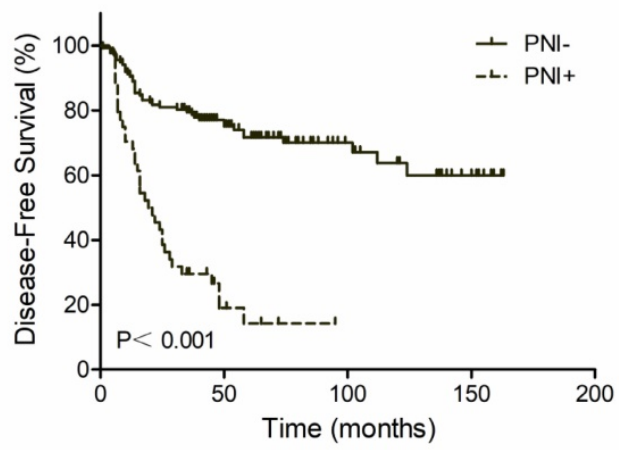

B

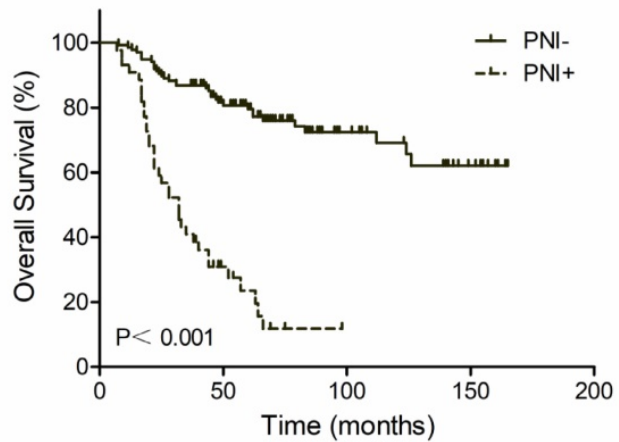

PNI or LVI is an independent prognostic factor of outcome in LARC patients

A Cox proportional hazard regression model was used to evaluate the influence of PNI, LVI, and other relevant covariates on survival. After controlling age, ypT stage, ypN stage, tumor distance from the anal verge, CEA level, and use of adjuvant chemotherapy, multivariate analysis indicated that PNI and LVI were independent prognostic factors for DFS (all $P$-value $<0.05$ ). PNI, rather than LVI, was also an independent prognostic factor for OS $(P<0.001)$ (Table 4). ypT stage and ypN stage also remained independent prognostic factors for DFS (all $P$-value $<0.05)$ and OS (all $P$-value<0.05) (Table 4).

Table 4. Multivariate analysis for disease-free and overall survival

\begin{tabular}{|c|c|c|c|c|}
\hline \multirow{2}{*}{$\begin{array}{l}\text { Multivariate } \\
\text { analysis }\end{array}$} & \multicolumn{2}{|c|}{ Disease-free survival } & \multicolumn{2}{|l|}{ Overall survival } \\
\hline & HR $(95 \%$ CI) & $P$ & HR $(95 \%$ CI) & $P$ \\
\hline \multicolumn{5}{|l|}{ PNI } \\
\hline $\begin{array}{l}\text { Positive vs. } \\
\text { negative }\end{array}$ & $2.115(1.163-3.848)$ & 0.014 & $2.936(1.669-5.164)$ & $<0.001$ \\
\hline \multicolumn{5}{|l|}{ LVI } \\
\hline $\begin{array}{l}\text { Positive vs. } \\
\text { negative }\end{array}$ & $1.932(1.096-3.405)$ & 0.023 & - & - \\
\hline \multicolumn{5}{|l|}{ ypT stage } \\
\hline T0-2 vs. T3-4 & 3.149 (1.614-6.143) & 0.001 & $3.006(1.538-5.876)$ & 0.001 \\
\hline \multicolumn{5}{|l|}{ ypN stage } \\
\hline N0 vs. N+ & $2.322(1.238-4.355)$ & 0.009 & $2.561(1.387-4.729)$ & 0.003 \\
\hline
\end{tabular}
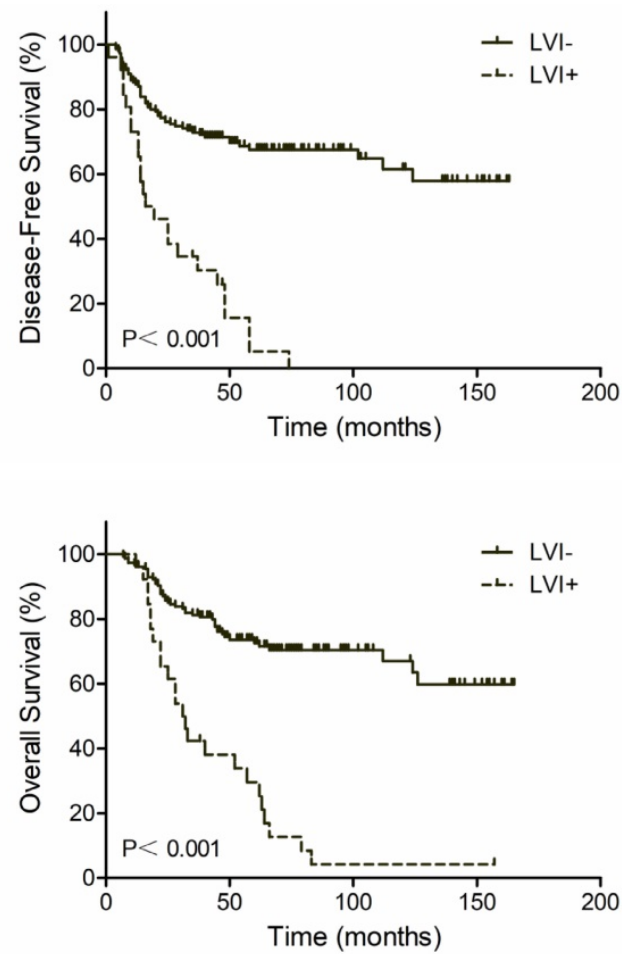

Figure 1. Kaplan-Meier survival curves according to perineural (PNI) and lymphovascular invasion (LVI). (A) DFS; (B) OS. 
A

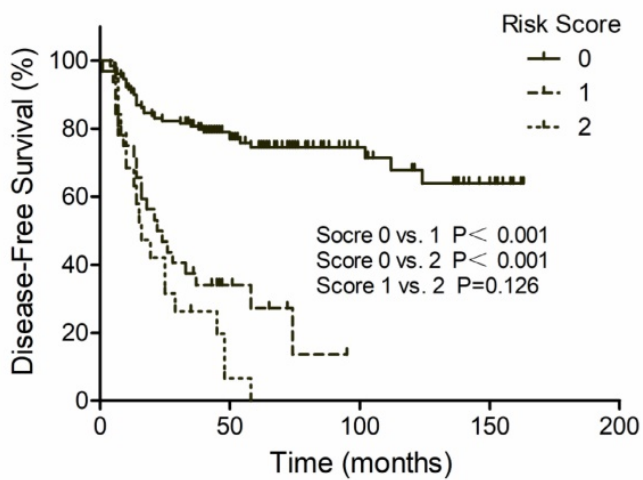

B

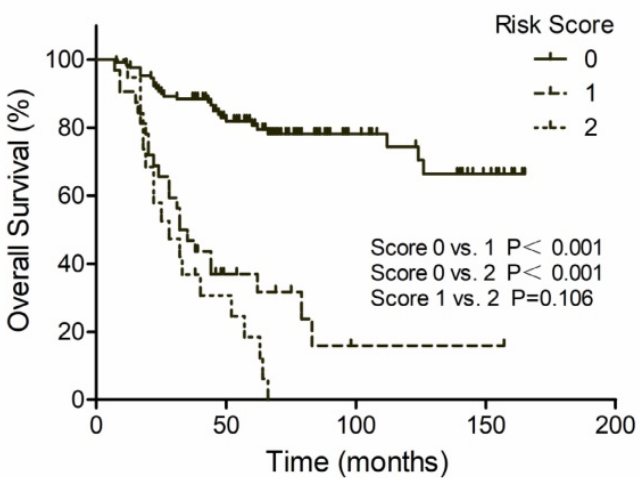

Figure 2. Kaplan-Meier survival curves according to risk score. (A) DFS; (B) OS. Score 0 represent both PNI and LVI negative; Score 1 represent PNI+/LVI- or PNI-/LVI+; Score 2 represent both $\mathrm{PNI}$ and LVI positive.

A

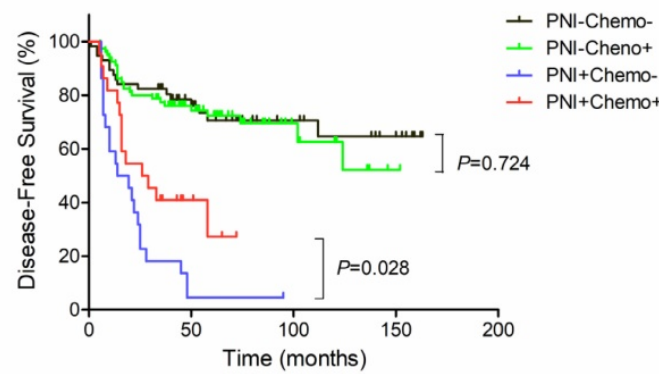

B

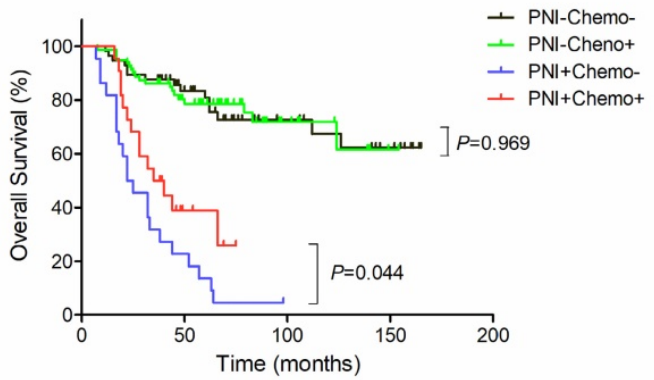

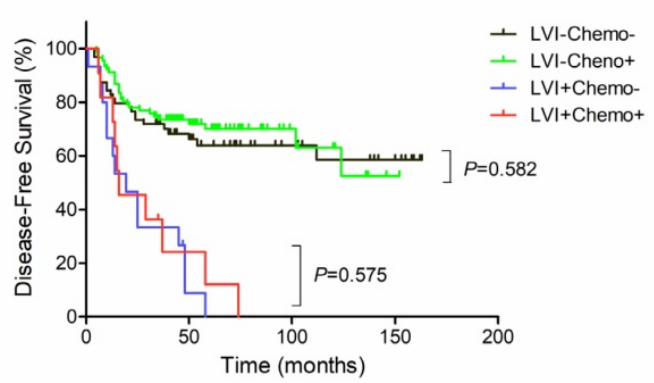

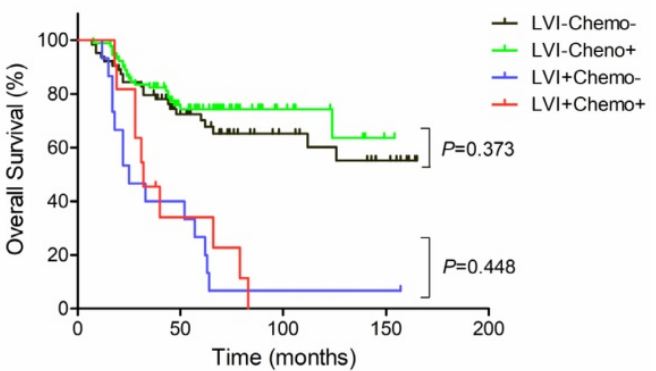

Figure 3. Kaplan-Meier survival curves according to PNI, LVI and adjuvant chemotherapy. (A) DFS; (B) OS.

\section{Adjuvant chemotherapy in a subgroup by PNI or LVI}

In PNI-positive subgroup, postoperative adjuvant chemotherapy improved 3-year DFS rate $(40.3 \%$ vs. $13.6 \%, P=0.028)$ and 3 -year OS rate $(39.5 \%$ vs. $22.7 \%, P=0.044$ ) (Figure $3 \mathrm{~A}$ and $3 \mathrm{~B}$, Left). In PNI-negative subgroup, no statistically significant differences were observed between with and without adjuvant chemotherapy in 3-year DFS $(75.9 \%$ vs. $78.2 \%, P=0.724)$ and 3 -year OS $(80.6 \%$ vs. $85.8 \%$, $P=0.969$ ) (Figure $3 \mathrm{~A}$ and $3 \mathrm{~B}$, Left). However, in LVI-positive subgroup, postoperative adjuvant chemotherapy did not significantly improve 3-year DFS rate $(23.6 \%$ vs. $25.9 \%, P=0.575)$ or 3 -year OS rate ( $35.4 \%$ vs. $40.0 \%, P=0.448$ ) (Figure $3 \mathrm{~A}$ and $3 \mathrm{~B}$, Right). Likewise, there were no statistically significant differences between with and without adjuvant chemotherapy in 3-year DFS $(73.5 \%$ vs. $68.0 \%$, $P=0.582)$ and 3 -year OS $(76.2 \%$ vs. $74.5 \%, P=0.373)$ in LVI-negative subgroup (Figure 3A and 3B, Right).

\section{Discussion}

In our study, we evaluated the significance of PNI and LVI as prognostic indicators in LARC after nCRT and surgery. Positive incisal margin, advanced ypT and ypN positive status were associated with PNI and LVI. Patients with PNI and/or LVI positive had a significantly higher risk of recurrence and poorer survival. Multivariate analysis indicated that PNI and LVI were independent prognostic factors of outcome in LARC patients. In addition, PNI-positive, rather than LVI-positive, may benefit from postoperative adjuvant chemotherapy. 
PNI refers to tumor invasion of the space surrounding a nerve and has been investigated in many cancers, including prostate, head and neck cancer, and colorectal cancer [10,19-21]. LVI is defined as tumor invasion in the vascular and lymphatic structure. Since it is infeasible to distinguish histologically between lymphatic and venous vessels, the term LVI usually refers to any of these structures [18]. In the current study, the incidence of PNI and LVI was $24.3 \%$ and $14.4 \%$, respectively, which is in-line with previous reports in preoperative CRT patients [22-24]. However, in untreated tumors, higher incidence of PNI and LVI of $30 \%$ and $70.6 \%$, respectively, were reported $[25,26]$. In these previous studies, multivariate analysis indicated that PNI or LVI was an independent negative prognosticator of survival. However, other two studies demonstrated that PNI had an impact on the DFS, but not the OS, based on multivariate analysis [11,27]. In our univariate analysis, we found that patients with PNI-positive or LVI-positive had poorer DFS and OS. In our multivariate analysis, PNI was a significant prognosticator affecting the DFS and OS, and LVI had an impact only on the DFS. In addition, subgroup analysis showed that in PNI-positive group, postoperative adjuvant chemotherapy improved DFS and OS. However, in LVI-positive group, postoperative adjuvant chemotherapy had no impact on DFS or OS. Lower incidence of LVI or the small sample size of our study may account for these results.

In our analysis, advanced ypT and ypN stage were significant factors associated with PNI and LVI in LARC after nCRT. However, studies have indicated that LVI was not significantly associated with the pT or $\mathrm{pN}$ stage in rectal cancer patients after preoperative radiotherapy rather than nCRT [28]. Thus, further studies may be conducted to address this controversial problem.

The present study has certain limitations, such as its retrospective nature, and therefore, the treatment delivery could not be controlled for the heterogeneity. Furthermore, the pathologists did not evaluate the pathologic specimens independently, which might lead to a potential error of evaluating PNI and LVI. Nevertheless, clinicopathologic and survival data were collected by reviewing the record of the individual patient. All patients were received standard treatment of nCRT and TME as recommended.

In summary, our study indicated that PNI and/or LVI following nCRT were poor prognosticators, and found the survival benefit of postoperative adjuvant chemotherapy to LARC patients with PNI.

\section{Acknowledgments}

This work was supported by the grants from the National Natural Science Foundation of China (NO. 81502646 and NO. 81502647), Zhejiang Provincial Natural Science Foundation of China (NO. LY14H160009), and the Zhejiang Medical and Health Science and Technology Project (NO. 2016KYB042 and NO. 2018KY298).

\section{Competing Interests}

The authors have declared that no competing interest exists.

\section{References}

1. van Gijn W, Marijnen CA, Nagtegaal ID, et al. Preoperative radiotherapy combined with total mesorectal excision for resectable rectal cancer: 12-year follow-up of the multicentre, randomised controlled TME trial. Lancet Oncol 2011; $12: 575-582$

2. Gerard JP, Conroy T, Bonnetain F, et al. Preoperative radiotherapy with or without concurrent fluorouracil and leucovorin in T3-4 rectal cancers: results of FFCD 9203. J Clin Oncol 2006; 24:4620-4625

3. Benson AR, Venook AP, Al-Hawary MM, et al. Rectal Cancer, Version 2.2018, NCCN Clinical Practice Guidelines in Oncology. J Natl Compr Canc Netw 2018; 16:874-901

4. Collette L, Bosset JF, den Dulk M, et al. Patients with curative resection of cT3-4 rectal cancer after preoperative radiotherapy or radiochemotherapy: does anybody benefit from adjuvant fluorouracil-based chemotherapy? A trial of the European Organisation for Research and Treatment of Cancer Radiation Oncology Group. J Clin Oncol 2007; 25:4379-4386

5. Valentini V, van Stiphout RG, Lammering G, et al. Nomograms for predicting local recurrence, distant metastases, and overall survival for patients with locally advanced rectal cancer on the basis of European randomized clinical trials. J Clin Oncol 2011; 29:3163-3172

6. Glynne-Jones R, Wyrwicz L, Tiret E, et al. Rectal cancer: ESMO Clinical Practice Guidelines for diagnosis, treatment and follow-up. Ann Oncol 2017; 28: v22-v40

7. Batsakis JG. Nerves and neurotropic carcinomas. Ann Otol Rhinol Laryngol 1985; 94:426-427

8. Compton CC, Fielding LP, Burgart LJ, et al. Prognostic factors in colorectal cancer. College of American Pathologists Consensus Statement 1999. Arch Pathol Lab Med 2000; 124:979-994

9. Cienfuegos JA, Rotellar F, Baixauli J, et al. Impact of perineural and lymphovascular invasion on oncological outcomes in rectal cancer treated with neoadjuvant chemoradiotherapy and surgery. Ann Surg Oncol 2015; 22:916-923

10. Liebig C, Ayala G, Wilks J, et al. Perineural invasion is an independent predictor of outcome in colorectal cancer. J Clin Oncol 2009; 27:5131-5137

11. Lee JH, Jang HS, Kim JG, et al. Lymphovascular invasion is a significant prognosticator in rectal cancer patients who receive preoperative chemoradiotherapy followed by total mesorectal excision. Ann Surg Oncol 2012; 19:1213-1221

12. Chablani $P$, Nguyen $P, P a n X$, et al. Perineural Invasion Predicts for Distant Metastasis in Locally Advanced Rectal Cancer Treated With Neoadjuvant Chemoradiation and Surgery. Am J Clin Oncol 2015

13. Ting WC, Chen LM, Huang LC, et al. Impact of interleukin-10 gene polymorphisms on survival in patients with colorectal cancer. J Korean Med Sci 2013; 28:1302-1306

14. Ptok $H$, Meyer $F$, Steinert $R$, et al. No prognostic impact of isolated lymphovascular invasion after radical resection of rectal cancer--results of a multicenter observational study. Int J Colorectal Dis 2007; 22:749-756

15. Fagan JJ, Collins B, Barnes L, et al. Perineural invasion in squamous cell carcinoma of the head and neck. Arch Otolaryngol Head Neck Surg 1998; 124:637-640

16. Bockman DE, Buchler M, Beger HG. Interaction of pancreatic ductal carcinoma with nerves leads to nerve damage. Gastroenterology 1994; 107:219-230

17. Peng J, Sheng W, Huang D, et al. Perineural invasion in pT3N0 rectal cancer: the incidence and its prognostic effect. Cancer-Am Cancer Soc 2011; 117:1415-1421

18. Hoda SA, Hoda RS, Merlin S, Shamonki J, Rivera M. Issues relating to lymphovascular invasion in breast carcinoma. Adv Anat Pathol 2006; 13:308-315

19. Beard $\mathrm{CJ}$, Chen $\mathrm{MH}_{\text {, }}$ Cote $\mathrm{K}$, et al. Perineural invasion is associated with increased relapse after external beam radiotherapy for men with low-risk prostate cancer and may be a marker for occult, high-grade cancer. Int J Radiat Oncol Biol Phys 2004; 58:19-24 
20. Harnden P, Shelley MD, Clements $H$, et al. The prognostic significance of perineural invasion in prostatic cancer biopsies: a systematic review. Cancer-Am Cancer Soc 2007; 109:13-24

21. Mendenhall WM, Amdur RJ, Hinerman RW, et al. Skin cancer of the head and neck with perineural invasion. Am J Clin Oncol 2007; 30:93-96

22. Ceyhan GO, Liebl F, Maak M, et al. The severity of neural invasion is a crucial prognostic factor in rectal cancer independent of neoadjuvant radiochemotherapy. Ann Surg 2010; 252:797-804

23. Poeschl EM, Pollheimer MJ, Kornprat P, et al. Perineural invasion: correlation with aggressive phenotype and independent prognostic variable in both colon and rectum cancer. J Clin Oncol 2010;28: e358-e360, e361-e362

24. Park IJ, You YN, Agarwal A, et al. Neoadjuvant treatment response as an early response indicator for patients with rectal cancer. J Clin Oncol 2012; 30:1770-1776

25. Liebig C, Ayala G, Wilks JA, Berger DH, Albo D. Perineural invasion in cancer: a review of the literature. Cancer-Am Cancer Soc 2009;115: 3379-3391

26. Sato $\mathrm{T}$, Ueno $\mathrm{H}$, Mochizuki $\mathrm{H}$, et al. Objective criteria for the grading of venous invasion in colorectal cancer. Am J Surg Pathol 2010; 34:454-462

27. Lim SB, Yu CS, Hong YS, et al. Long-term outcomes in patients with locally advanced rectal cancer treated with preoperative chemoradiation followed by curative surgical resection. J Surg Oncol 2012;106: 659-666

28. Ptok H, Meyer F, Steinert $R$, et al. No prognostic impact of isolated lymphovascular invasion after radical resection of rectal cancer--results of a multicenter observational study. Int J Colorectal Dis 2007; 22:749-756 